\title{
Metal organic vapour phase epitaxial growth of indium-rich InGaN alloys with robust photoluminescence properties
}

\author{
M. Moret, S. Ruffenach, O. Briot, and B. Gil ${ }^{\mathrm{a}}$ \\ Université Montpellier 2, Groupe d'Étude des Semiconducteurs, UMR-CNRS 5650, Case Courrier 74, 34095 Montpellier \\ Cedex 5, France
}

Received: 11 September 2008 / Accepted: 11 December 2008

Published online: 31 January 2009 - (c) EDP Sciences

\begin{abstract}
We report the growth of indium-rich InGaN alloys by metal-organic vapour phase epitaxy, using ammonia, trimethylgallium and trimethylindium as precursors. Compared to indium nitride, our alloy samples present substantial photoluminescence robustness with temperature. The analysis of the optical properties of these samples versus temperature indicates the existence of two non radiative recombination channels: one with a thermal activation temperature of $77 \mathrm{~K}$ and another one with an activation temperature ranging from $300 \mathrm{~K}$ for $\mathrm{InN}$ up to $640 \mathrm{~K}$ for $\operatorname{In}_{0.72} \mathrm{Ga}_{0.28} \mathrm{~N}$. The latter competes with the former at low temperatures whilst the former rules the optical properties at ambient conditions.
\end{abstract}

PACS. 78.20.-e Optical properties of bulk materials and thin films - 78.55.-m Photoluminescence, properties and materials - 71.55.Eq III-V semiconductors

\section{Introduction}

Indium nitride and related heterostructures are promising materials for realizing arsenic-free optoelectronic devices operating in the near infrared portion of the electromagnetic spectrum. The band gap of indium nitride sits at $650 \mathrm{meV}$ [1] and recent demonstrations using the molecular beam epitaxy technique of $\mathrm{InN}$ based quantum well heretostructures with quantum confined stark effect [2-4] indicate potential applications of these materials in various field as optical modulators, optical switches and other light emitting/detecting devices. In this paper we report the growth of indium-rich InGaN layers by metal organic vapour phase epitaxy with photoluminescence properties being robust with temperature as a probe of a fairly reasonable density of non radiative photoluminescence killing centres.

\section{Samples growth}

The samples were grown by low pressure metal-organic vapour phase epitaxy, using an AIXTRON AIX200/4RF$\mathrm{S}$ reactor. The growth temperature was $550{ }^{\circ} \mathrm{C}$ and reactor pressure 200 mbar for all samples, with a molar V/III ratio of 15000 , in order to prevent any indium droplet formation. Trimethylindium (TMIn) and trimethylgallium (TMGa), provided by SAFC Hitech Limited, were used as

\footnotetext{
a e-mail: gil@ges.univ-montp2.fr
}

group III element precursors, ammonia $\left(\mathrm{NH}_{3}\right)$ was the nitrogen source. The InGaN layers were grown using $\mathrm{N}_{2}$ as carrier gas, since hydrogen damages InN, but hydrogen was used as carrier gas for GaN. The purification of $\mathrm{H}_{2}$ and $\mathrm{N}_{2}$, as well as $\mathrm{NH}_{3}$ was ensured by a complete SAES setup of corresponding purifiers. The different Indium contents were obtained by changing the TMIn/TMGa ratio without changing the total molar flow. The growth rate of InGaN is insensitive to Ga content due to the similar low growth rate of both binaries, which are limited by the low decomposition rate of ammonia. The (In, Ga)N layers thicknesses were measured by SEM and are about $800 \mathrm{~nm}$ for all the samples.

The InGaN layers were deposited on top of a GaN epilayer (typically $1 \mu \mathrm{min}$ thickness), deposited onto a sapphire substrate. X-ray rocking curve measurements were performed on GaN pseudo-substrate and indicate comparable defects densities in all of them. The samples were characterized by X-ray diffraction to determine their composition and crystalline quality, the theta/2theta measurement are displayed Figure 1.

\section{Photoluminesnence results and modelisation}

In Figure 2 is reported the evolution of the photoluminescence intensity of an $\mathrm{In}_{0.72} \mathrm{Ga}_{0.28} \mathrm{~N}$ layer for temperatures ranging from 4 to $300 \mathrm{~K}$. An extended InGaAs Detector was used together with a grating blazed at $1.5 \mu \mathrm{m}$. 


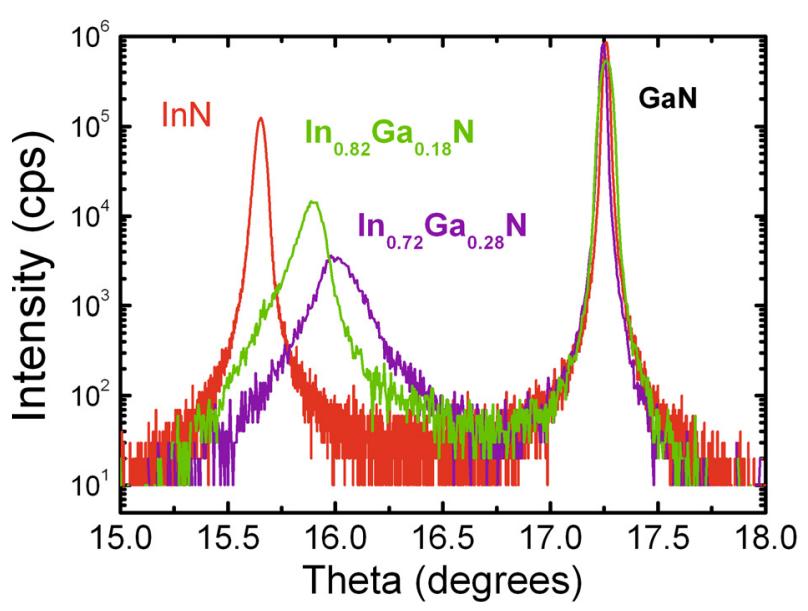

Fig. 1. (Color online) Theta-2theta X-ray diffraction features.

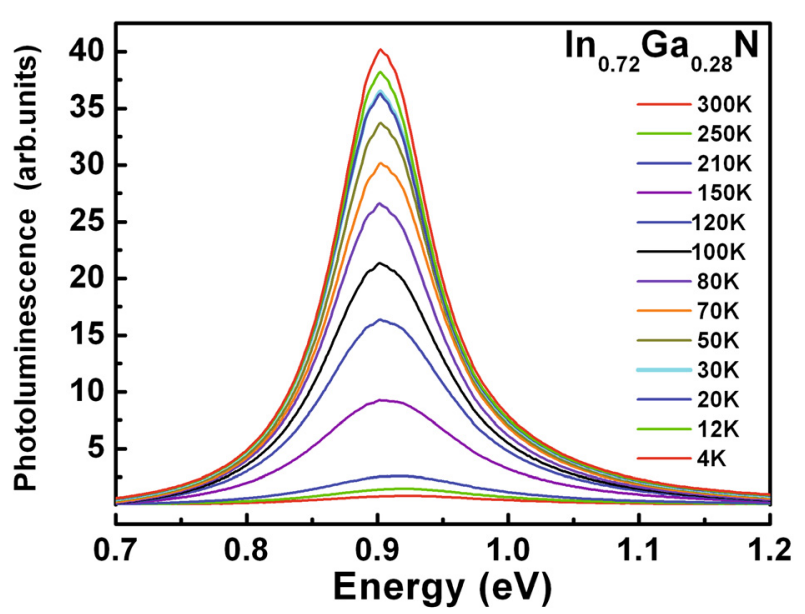

Fig. 2. (Color online) Evolution of the photoluminescence spectra of $\operatorname{In}_{0.72} \mathrm{Ga}_{0.28} \mathrm{~N}$ layers with temperature.

The trend is a collapse of the photoluminescence intensity with increasing temperature, as a probe of the presence of non radiative recombination channels in this sample and in all others of ours. The figure shows the robustness of the intensity of the photoluminescence which in case of $\mathrm{In}_{0.72} \mathrm{Ga}_{0.28} \mathrm{~N}$ layers decreases by a factor 30 between $4 \mathrm{~K}$ and room temperature. This collapse scales two decades for high quality InN films. We have recorded the evolution of the energy of the photoluminescence peaks with temperature. We find a red shift of $59 \mathrm{meV}$ and $1 \mathrm{meV}$ for $\mathrm{InN}$ and $\mathrm{In}_{0.82} \mathrm{Ga}_{0.18} \mathrm{~N}$ layers respectively. In contrast to this, we report observation of a $20 \mathrm{meV}$ blue shift for $\mathrm{In}_{0.72} \mathrm{Ga}_{0.28} \mathrm{~N}$ layer. The $\mathrm{InN}$ value is comparable with the value reported for best samples in the literature [1], to the best of our knowledge. Our InN data indicates an initial redshift up to $50 \mathrm{~K}$ then a weak blue shift and a general trend that is easily fitted using a Varshni equation. This weak $S$-shape behaviour is an indication of the weakly localized nature of the excitons or $e-h$ pairs [5] which recombine at low temperature and of a bound to free phase transition near $60 \mathrm{~K}$. Concerning the alloys, we are probing the more complex physics of recombina- tion of bound or localized excitons or $e-h$ pairs in strongly disordered systems. It is worthwhile noticing in particular that a simple line shape fitting of the photoluminescence lines performed in order to obtain the maximum of fluorescence energy indicates the onset of blue shift of the photoluminescence line for the 28-percent gallium-rich sample to occurs near 100-120 K ( 10 meV activation energy) simultaneously when the photoluminescence intensity starts to decrease. We attribute this behaviour to simultaneous detrapping of excitons (or photo-created carriers) from shallow low energy potential fluctuations lakes surrounded by barriers of moderate heights and to their further retrapping to other higher energy regions of the crystal surrounded by higher potential barriers. This behaviour is different from the behaviour that is generally reported for gallium-rich InGaN alloys. There are several reasons to this. First, the value of the band gap bowing parameter is large for InGaN alloys $(2.8 \mathrm{eV})$ [6-9]. Fluctuations of the chemical alloy composition give deeper wells for gallium rich alloys than they give for indium-rich ones. The spontaneous production of shallow potential wells by strict chemical disorder is more favourable in case of indium rich alloys than for gallium rich ones. Second, the input parameters used to describe the physics of these materials in the context of quantum mechanics are very different.

This reflects the competition between the average spatial extension of the chemical disorder induced potential fluctuations and the spatial extension of carrier (electrons and holes) wavefunction.

Let us restrict ourselves here to electrons, for the sake of our discussion. The effective mass is very small for InN (0.07) while it is substantially high (0.22) for GaN [1]. Let us consider twenty percent alloy disorders near both ends of the full composition range. Effective mass arguments tell us that an electron can probe a more extended crystal region in the case of an indium-rich alloy than it would do it in case of a gallium rich one. Therefore the carrier trapping - detrapping processes are a priori more complicated for indium-rich alloys than they are for gallium-rich ones. This will be inferred later in this paper. We now examine the behaviour of the emitted photoluminescence intensity with increasing temperature. In the most general way, the photoluminescence intensity of excitons or $e-h$ pairs is given by the following proportionality relation:

$$
I \infty \frac{\tau}{\tau_{r a d}}
$$

where $\tau$ is the photoluminescence decay time, $\tau_{\text {rad }}$ is the radiative photoluminescence decay time. Further introducing the non radiative part $\tau_{n r}$ of the decay time in equation (1) we get:

$$
I(T) \infty \frac{\tau_{n r}}{\tau_{n r}+\tau_{r a d}}=\frac{1}{1+\frac{\tau_{r a d}}{\tau_{n r}}} .
$$

Statistical physics arguments tell us that $\tau_{\text {rad }}$ is proportional to $T^{n / 2}$ where $\mathrm{n}$ is the dimensionality of the crystal. Integer $n=3$ in a bulk, 2 for a quantum well, 1 for a quantum wire and 0 for a zero dimensional confining centre 
Table 1. Summary of the fitting procedure and correlation function between the fit and the data.

\begin{tabular}{|c|c|c|c|c|c|}
\hline & $A$ & $T_{0}(\mathrm{~K})$ & $B$ & $T_{1}(\mathrm{~K})$ & Cor \\
\hline $\operatorname{InN}$ & 5 & $77 \pm 3$ & $93 \pm$ & 16 & 0.99958 \\
\hline $\operatorname{In}_{0.8}$ & $2.4 \pm 0.3$ & $77 \pm 5$ & $66 \pm 15$ & $400 \pm 30$ & 0.9 \\
\hline $\mathrm{n}_{0.72} \mathrm{Ga}_{0.28} \mathrm{~N}$ & $0.8 \pm 0.1$ & $78 \pm 7$ & $156 \pm 31$ & $638 \pm 30$ & 0.99801 \\
\hline
\end{tabular}

(quantum dot or 3D localizing centre). The non radiative recombination channels (we supposed to have several ones) are thermally activated:

$$
\frac{1}{\tau_{n r}}(T)=\sum_{i} \frac{1}{\tau_{i}}(T) .
$$

In general, the evolution of photoluminescence intensity is fitted in the context of an Arrhenius plot, that is to say by plotting the logarithm of the intensity versus the reciprocal temperature in order to obtain in a more or less readable manner, from the intensity behaviour at high temperatures, an activation temperature $T_{i}$ that rules the non radiative recombination channel $i$. This gives a rough estimate of what happens. A complementary approach consists in adding to this a fit of the linear dependence of the photoluminescence intensity in order to correctly describe the complex statistics physics in the range of temperatures where radiative and non radiative recombination channels do compete. From the behaviour of the sample with 28-percent gallium we immediately remark the clear need to introduce two non radiative recombination channels in order to fit the experiment. This means that we have to handle the following equation:

$$
I(T) \infty \frac{1}{1+A e^{\frac{T_{0}}{T}}+B e^{\frac{T_{1}}{T}},}
$$

where $A, B, T_{0}$ and $T_{1}$ are fitting parameters.

Table 1 indicates the result of the full fitting procedure. The remarkable result is the constant value we get for $T_{0}=77 \mathrm{~K}$ which correspond to an activation energy of some $6.6 \mathrm{meV}$. It is worthwhile noticing that this weak activation energy corresponds to the detrapping energy we always observe in our InN samples. Next we remark that $T_{1}$ increases with the alloy composition from $300 \mathrm{~K}$ $(26 \mathrm{meV})$ for $\mathrm{InN}$ up to $640 \mathrm{~K}(55 \mathrm{meV})$ for $\operatorname{In}_{0.72} \mathrm{Ga}_{0.28} \mathrm{~N}$.

In Figures 3 and 4 are reported the results of the fitting procedures in the context of the reciprocal and direct temperatures respectively. A complementary plot which gives the evolution of the ratio: $(B / A) e^{\left(T_{1}-T_{0}\right) / T}$ for these samples is shown in Figure 5 where one can clearly see that the robustness of the photoluminescence in InGaN layers is correlated with the high values reached by this ratio at high temperatures: the relative values of $A$ and $B$ are such that the low temperature $T_{0}$ activated process does not dominate. Although it is difficult to make some more deterministic physics we have to remark in Table 1 the large values of $B$ and their increase with increasing the alloy composition. This clearly indicates (having in mind that $\tau_{\text {rad }}$ is essentially a function of material

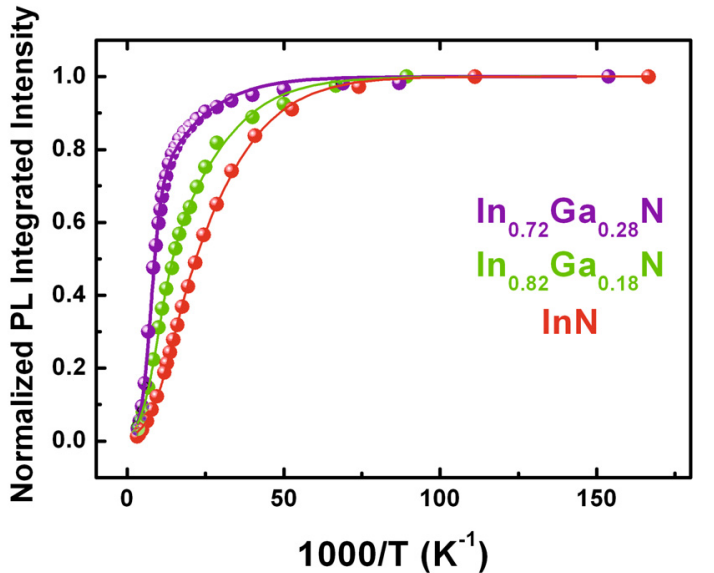

Fig. 3. (Color online) Plot of the photoluminescence intensity versus reciprocal temperature.

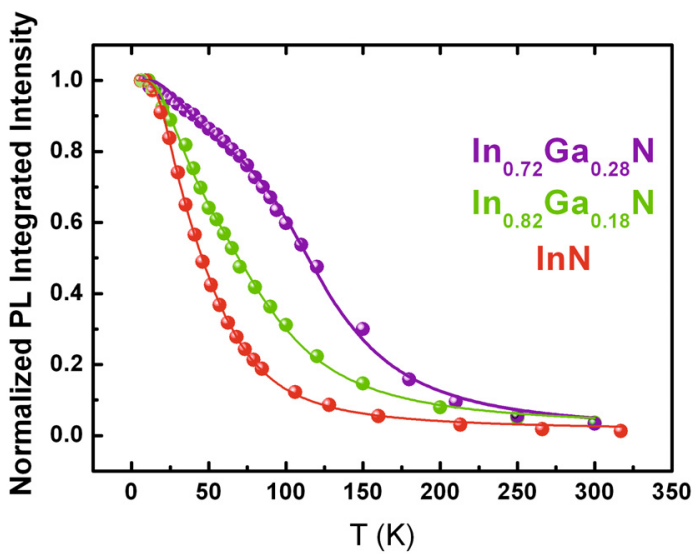

Fig. 4. (Color online) Plot of the photoluminescence intensity versus temperature.

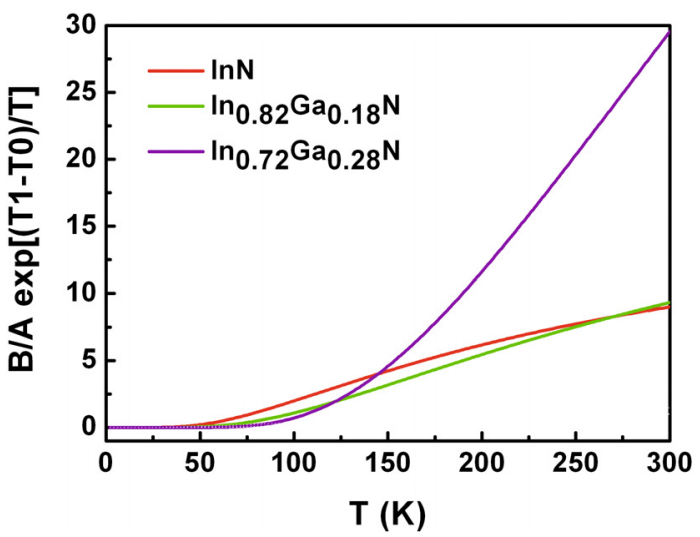

Fig. 5. (Color online) Relative amplitudes of the non radiative recombination channels.

parameters like the effective masses, the dielectric constant,...; that is to say having in mind that $\tau_{\text {rad }}$ does not vary so much in these samples), that the characteristics non radiative decay time $\tau_{1}$ decreases when increasing the alloy composition. This is consistent with:

(i) our X-ray diffraction patterns which are slightly worse for the alloy than it is for the binary compound as 
an indication of an - increasing number of topological defects with increasing the alloy composition and;

(ii) the behaviour of the absolute intensities of the photoluminescence features taken at $4 \mathrm{~K}$ which decrease with increasing the alloy composition.

\section{Conclusion}

In conclusion, this work reports the promising observation of the robustness of the photoluminescence intensity with temperature for indium-rich InGaN alloys grown by metal organic vapour phase epitaxy (as far as we are aware the materials produced by molecular beam epitaxy do not exhibit this robustness). This demonstration indicates that this growth technique has reached a sufficient degree of maturity to provide samples with interesting optical properties in a near future. Further and unavoidable improvements of the crystalline quality of these materials will then open the way towards realization of heterostructures based on $\mathrm{InN}$ and related alloys, with remarkable and broad fields of application in the near infrared region.

This work was supported by the European Commission under the Sixth Framework Program (STREP project "INDOT", Contract No. NMP4-CT-2005-016956).

\section{References}

1. For a general review, see for instance: W. Walukiewicz, S.X. Li, J. Wu, K.M. Yu, J.W. Ager, E.E. Haller, H. Lu, W.J. Schaff, J. Cryst. Growth 269, 119 (2004)

2. M. Kurouchi, H. Naoi, T. Araki, T. Miyajima, Y. Nanishi, Jpn J. Appl. Phys. 44, L230 (2005)

3. T. Onashi, P. Holmström, A. Kikuchi, K. Kishino, Appl. Phys. Lett. 89, 041907 (2006)

4. S.-B. Che, T. Minuno, X. Wang, Y. Ishitani, A. Yoshikawa, J. Appl. Phys. 102, 083539 (2007)

5. S. Chichibu, Y. Kawakami, T. Soda, in Introduction to nitride semiconductor blue lasers and light emitting diodes, edited by S. Nakamura, S. Chichibu (Taylor and Francis, New york, 2000), p. 153, ISBN 0-7484-0836-3

6. V.Y. Davydov, A.A. Klochikhin, V.V. Emtsev, S.V. Ivanov, V.V. Vekshin, F. Bechstedt, J. Furthmuller, H. Harima, A.V. Mudryi, A. Hashimoto, A. Yamamoto, J. Aderhold, J. Graul, E.E. Haller, Phys. Stat. Sol. (b) 230, R42 (2002)

7. S. Pereira, M.R. Correia, T. Monteiro, E. Pereira, E. Alves, A.D. Sequeira, N. Franco, Appl. Phys. Lett. 78, 2137 (2001)

8. W. Shan, W. Walukiewicz, E.E. Haller, B.D. Little, J.J. Song, M.D. McCluskey, N.M. Johnson, Z.C. Feng, M. Schurman, R.A. Stall, J. Appl. Phys. 84, 4452 (1998)

9. J. Wu, W. Walukiewicz, K.M. Yu, J.W. Ager III, E.E. Haller, H. Lu, W.J. Schaff, Appl. Phys. Lett. 80, 4741 (2002) 